
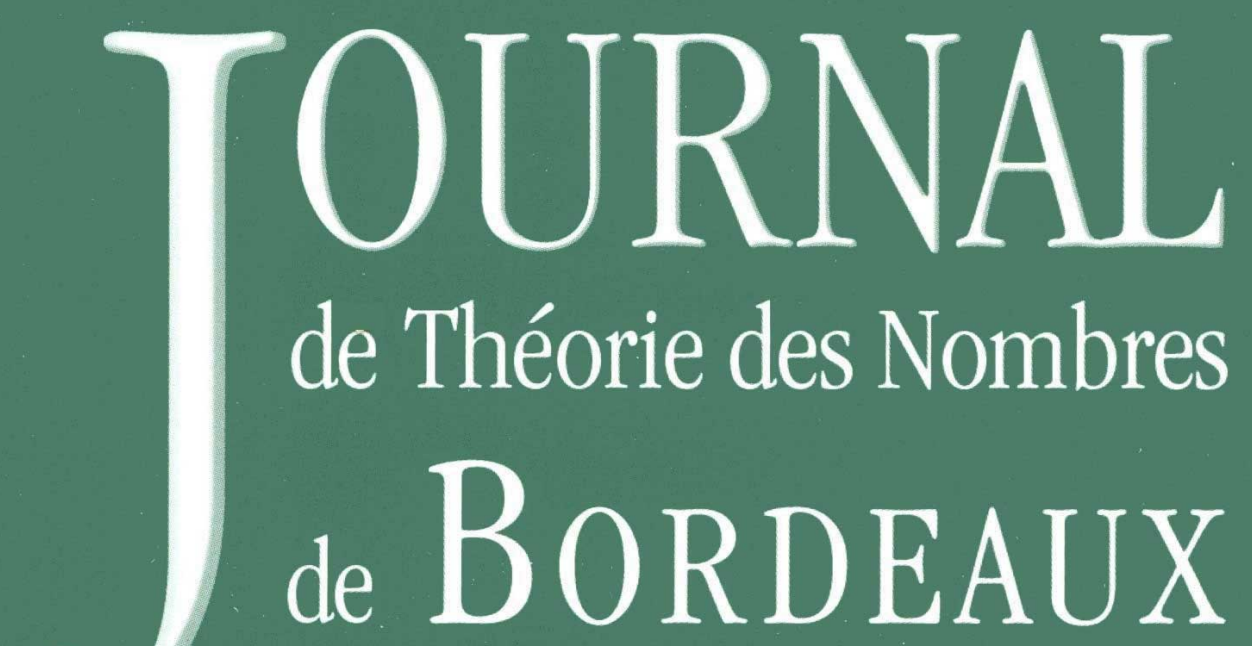

anciennement Séminaire de Théorie des Nombres de Bordeaux

\title{
Simon KRISTENSEN
}

Badly approximable systems of linear forms over a field of formal series

Tome 18, no 2 (2006), p. 421-444.

<http://jtnb.cedram.org/item?id=JTNB_2006__18_2_421_0>

(C) Université Bordeaux 1, 2006, tous droits réservés.

L'accès aux articles de la revue «Journal de Théorie des Nombres de Bordeaux » (http://jtnb.cedram.org/), implique l'accord avec les conditions générales d'utilisation (http://jtnb.cedram. org/legal/). Toute reproduction en tout ou partie cet article sous quelque forme que ce soit pour tout usage autre que l'utilisation à fin strictement personnelle du copiste est constitutive d'une infraction pénale. Toute copie ou impression de ce fichier doit contenir la présente mention de copyright.

\section{cedram}




\title{
Badly approximable systems of linear forms over a field of formal series
}

\author{
par SIMON KRISTENSEN
}

\begin{abstract}
RÉSumÉ. Nous montrons que la dimension de Hausdorff de l'ensemble des systèmes mal approchables de $m$ formes linéaires en $n$ variables sur le corps des séries de Laurent à coefficients dans un corps fini est maximale. Ce résultat est un analogue de la généralisation multidimensionnelle de Schmidt du théorème de Jarník sur les nombres mal approchables.
\end{abstract}

Abstract. We prove that the Hausdorff dimension of the set of badly approximable systems of $m$ linear forms in $n$ variables over the field of Laurent series with coefficients from a finite field is maximal. This is an analogue of Schmidt's multi-dimensional generalisation of Jarník's Theorem on badly approximable numbers.

\section{Introduction}

Let $\mathbb{F}$ denote the finite field of $q=p^{r}$ elements, where $p$ is a prime and $r$ is a positive integer. We define

$$
\mathcal{L}=\left\{\sum_{i=-n}^{\infty} a_{-i} X^{-i}: n \in \mathbb{Z}, a_{i} \in \mathbb{F}, a_{n} \neq 0\right\} \cup\{0\} .
$$

Under usual addition and multiplication, this set is a field, sometimes called the field of formal Laurent series with coefficients from $\mathbb{F}$ or the field of formal power series with coefficients from $\mathbb{F}$. An absolute value $\|\cdot\|$ on $\mathcal{L}$ can be defined by setting

$$
\left\|\sum_{i=-n}^{\infty} a_{-i} X^{-i}\right\|=q^{n}, \quad\|0\|=0 .
$$

Under the induced metric, $d(x, y)=\|x-y\|$, the space $(\mathcal{L}, d)$ is a complete metric space. Furthermore the absolute value satisfies for any $x, y \in \mathcal{L}$,

$$
\|x+y\| \leq \max (\|x\|,\|y\|) .
$$

Manuscrit reçu le $1^{\text {er }}$ septembre 2004. 
Property (1.2) is known as the non-Archimedean property. In fact, equality holds in (1.2) whenever $\|x\| \neq\|y\|$.

As we will be working in finite dimensional vector spaces over $\mathcal{L}$, we need an appropriate extension of the one-dimensional absolute value.

Definition. Let $h \in \mathbb{N}$. For any $\mathbf{x}=\left(x_{1}, \ldots, x_{h}\right) \in \mathcal{L}^{h}$, we define the height of $x$ to be

$$
\|\mathbf{x}\|_{\infty}=\max \left\{\left\|x_{1}\right\|, \ldots,\left\|x_{h}\right\|\right\}
$$

It is straightforward to see that (1.2) holds for $\|\cdot\|_{\infty}$. Of course, when $h=1$, this is the usual absolute value, and as in the one-dimensional case, $\|\cdot\|_{\infty}$ induces a metric on $\mathcal{L}^{h}$. When we speak of balls in any of the spaces $\mathcal{L}^{h}$, we will mean balls in this metric. Note that topologically, balls in $\mathcal{L}^{h}$ are both closed and open. All balls in this paper will be defined by large inequality (rather than strict inequality).

An important consequence of (1.2) is that if $C_{1}$ and $C_{2}$ are balls in some space $\mathcal{L}^{h}$, then either $C_{1} \cap C_{2}=\emptyset, C_{1} \subseteq C_{2}$ or $C_{2} \subseteq C_{1}$. We will refer to this property as the ball intersection property.

In $\mathcal{L}$, the polynomial ring $\mathbb{F}[X]$ plays a rôle analogous to the one played by the integers in the field of real numbers. Thus, we define the polynomial part of a non-zero element by

$$
\left[\sum_{i=-n}^{\infty} a_{-i} X^{-i}\right]=\sum_{i=-n}^{0} a_{-i} X^{-i}
$$

whenever $n \geq 0$. When $n<0$, the polynomial part is equal to zero. Likewise, the polynomial part of the zero element is itself equal to zero. We define the set

$$
I=\{x \in \mathcal{L}:\|x\| \leq 1\}
$$

the unit ball in $\mathcal{L}$.

With the above definitions, it makes sense to define the distance to $\mathbb{F}[X]^{h}$ from a point $\mathbf{x} \in \mathcal{L}^{h}$ :

$$
|\langle\mathbf{x}\rangle|=\min _{\mathbf{p} \in \mathbb{F}[X]^{h}}\|\mathbf{x}-\mathbf{p}\|_{\infty}
$$

Since we will be concerned with matrices, we let $m, n \in \mathbb{N}$ be fixed throughout the paper. We will need a number of unspecified constants which may depend on $m$ and $n$. To avoid cumbersome notation, for such constants, we will only specify the dependence on parameters other than $m$ and $n$.

We identify the $m \times n$-matrices with coefficients from $\mathcal{L}$ with $\mathcal{L}^{m n}$ in the usual way. Matrix products and inner products are defined as in the real case. Matrices will be denoted by capital letters, whereas vectors will be denoted by bold face letters. 
In this paper, we are concerned with the Hausdorff dimension (defined below) of the set of badly approximable systems of linear forms over $\mathcal{L}$, defined as follows.

Definition. Let $m$ and $n$ be positive integers. The set of matrices $\mathfrak{B}(m, n)$ is defined to be the set of all $A \in \mathcal{L}^{m n}$ for which there is a $K=K(A)>0$ such that for any $\mathbf{q} \in \mathbb{F}[X]^{m} \backslash\{0\}$,

$$
|\langle\mathbf{q} A\rangle|^{n}>\frac{K}{\|\mathbf{q}\|_{\infty}^{m}}
$$

This set is called the set of badly approximable elements in $\mathcal{L}^{m n}$.

On taking $n$ 'th roots on either side of the defining inequality, we see that the exponent of $\|\mathbf{q}\|_{\infty}$ on the right hand side becomes $m / n$. This is exactly the critical exponent in the Laurent series analogue of the KhintchineGroshev theorem [5, Theorem 1]. It is natural to suspect that an analogue of Dirichlet's theorem exists, and indeed this is the case.

Theorem 1.1. Let $A \in \mathcal{L}^{m n}$ and let $Q \geq 0$ be an integer with $n \mid Q m$. There is a $\mathbf{q} \in \mathbb{F}[X]^{m}$ with $\|\mathbf{q}\|_{\infty} \leq q^{Q}$, such that

$$
|\langle\mathbf{q} A\rangle|^{n} \leq \frac{1}{q^{Q m}} .
$$

We will deduce this theorem from work of Mahler [7]. Alternative proofs may be given, see e.g. the Proposition of Appendix 1 of [2]. Here it is shown that the enumerator on the right hand side of the inequality in Theorem 1.1 may be replaced by $q^{-1}$ for $m=n=1$ under the additional assumption that $\|A\| \leq 1$. Under those assumptions, this is best possible.

Let $\mu$ denote the Haar measure on $\mathcal{L}^{m n}$, i.e., the $m n$-fold product measure of the Haar measure on $\mathcal{L}$ (also denoted by $\mu$ ), which is characterised by

$$
\mu\left(\left\{x \in \mathcal{L}:\|x-c\| \leq q^{r}\right\}\right)=q^{r} .
$$

It is an easy consequence of $[5$, Theorem 1$]$ that $\mathfrak{B}(m, n)$ is a null-set, i.e., $\mu(\mathfrak{B}(m, n))=0$, for any $m, n \in \mathbb{N}$. This raises the natural question of the Hausdorff dimension of $\mathfrak{B}(m, n)$, which is shown to be maximal (Theorem 1.2 below), thus proving an analogue of Schmidt's Theorem on badly approximable systems of linear forms over the real numbers [10]. Niederreiter and Vielhaber [8] proved using continued fractions that $\mathfrak{B}(1,1)$ has Hausdorff dimension 1, i.e., a formal power series analogue of Jarník's Theorem [4]. The $p$-adic analogue of Jarník's Theorem was proven by Abercrombie $[1]$.

Hausdorff dimension in this setting is defined as follows: Let $E \subseteq \mathcal{L}^{m n}$. For any countable cover $\mathcal{C}$ of $E$ with balls $B_{i}=B\left(\mathbf{c}_{i}, \rho_{i}\right)$, we define the 
$s$-length of $\mathcal{C}$ as the sum

$$
l^{s}(\mathcal{C})=\sum_{B_{i} \in \mathcal{C}} \rho_{i}^{s}
$$

for any $s \geq 0$. Restricting to covers $\mathcal{C}_{\delta}$, such that for some $\delta>0$, any ball $B_{i} \in \mathcal{C}_{\delta}$ has radius $\rho_{i}<\delta$, we can define an outer measure

$$
\mathcal{H}^{s}(E)=\lim _{\delta \rightarrow 0} \inf _{\text {covers }} \mathcal{C}_{\delta} l^{s}\left(\mathcal{C}_{\delta}\right),
$$

commonly called the Hausdorff $s$-measure of $E$. It is straightforward to prove that this is indeed an outer measure. Also, given a set $E \subseteq \mathcal{L}^{m n}$, the Hausdorff $s$-measure of $E$ is either zero or infinity for all values of $s \geq 0$, except possibly one. Furthermore, the Hausdorff $s$-measure of a set is a non-increasing function of $s$. We define the Hausdorff $\operatorname{dimension} \operatorname{dim}(E)$ of a set $E \subseteq \mathcal{L}^{m n}$ by

$$
\operatorname{dim}(E)=\inf \left\{s \geq 0: \mathcal{H}^{s}(E)=0\right\} .
$$

As in the real case, it can be shown that $\operatorname{dim}(E) \leq m n$ for any $E \subseteq \mathcal{L}^{m n}$.

With these definitions, we prove

Theorem 1.2. Let $m$ and $n$ be positive integers. Then,

$$
\operatorname{dim}_{\mathrm{H}}(\mathfrak{B}(m, n))=m n .
$$

We will use the method developed by Schmidt [9] to prove the analogous one-dimensional real result, namely the so-called $(\alpha, \beta)$-games. Schmidt [10] subsequently used this method to prove the multi-dimensional real analogue of Theorem 1.2.

The rest of the paper is organised as follows. In section 2 , we define $(\alpha, \beta)$ games and some related concepts and state some results due to Mahler [7] from the appropriate analogue of the geometry of numbers in the present setting. We will also deduce Theorem 1.1 from the results of Mahler.

The $(\alpha, \beta)$-game has two players, White and Black, with parameters $\alpha$ and $\beta$ respectively. When played, the game terminates after infinitely many moves in a single point in the space $\mathcal{L}^{m n}$. We prove in section 3 , that for $\alpha$ small enough, player White may ensure that the point in which the game terminates is an element of $\mathfrak{B}(m, n)$. The fundamental tools in this proof are a transference principle and a reduction of the statement to a game which terminates after a finite number of moves. The transference principle allows us to use the approximation properties of a matrix to study the approximation properties of the transpose of the same matrix. The finite game allows us to show that player White may ensure that all the undesirable points with $\|\mathbf{q}\|_{\infty}$ less than an appropriate bound can be avoided. This is the most extensive part of the paper, and the proof is quite technical. 
Finally, in section 4, we use the property from section 3 to show that the dimension of $\mathfrak{B}(m, n)$ must be greater than or equal to $m n$. Together with the above remarks, this implies Theorem 1.2.

\section{Notation, definitions and preliminary results}

We now define $(\alpha, \beta)$-games, which will be our main tool in the proof of Theorem 1.2. Let $\Omega=\mathcal{L}^{m n} \times \mathbb{R}_{\geq 0}$. We call $\Omega$ the space of formal balls in $\mathcal{L}^{m n}$, where $\omega=(\mathbf{c}, \rho) \in \Omega$ is said to have centre $\mathbf{c}$ and radius $\rho$. We define the map $\psi$ from $\Omega$ to the subsets of $\mathcal{L}^{m n}$, assigning a real $\|\cdot\|_{\infty}$-ball to the abstract one defined above. That is, for $\omega=(\mathbf{c}, \rho) \in \Omega$,

$$
\psi(\omega)=\left\{\mathbf{x} \in \mathcal{L}^{m n}:\|\mathbf{x}-\mathbf{c}\|_{\infty} \leq \rho\right\} .
$$

Definition. Let $B_{1}, B_{2} \in \Omega$. We say that $B_{1}=\left(\mathbf{c}_{1}, \rho_{1}\right) \subseteq B_{2}=\left(\mathbf{c}_{2}, \rho_{2}\right)$ if $\rho_{1}+\left\|\mathbf{c}_{1}-\mathbf{c}_{2}\right\|_{\infty} \leq \rho_{2}$.

Note that if $B_{1} \subseteq B_{2}$ in $\Omega$, then $\psi\left(B_{1}\right) \subseteq \psi\left(B_{2}\right)$ as subsets of $\mathcal{L}^{m n}$. Also, we define for every $\gamma \in(0,1)$ and $B \in \Omega$ :

$$
B^{\gamma}=\left\{B^{\prime} \subseteq B: \rho\left(B^{\prime}\right)=\gamma \rho(B)\right\} \subseteq \Omega,
$$

where $\rho(B)$ is the radius of $B$. We now define the following game.

Definition. Let $S \subseteq \mathcal{L}^{m n}$, and let $\alpha, \beta \in(0,1)$. Let Black and White be two players. The $(\alpha, \beta ; S)$-game is played as follows:

- Black chooses a ball $B_{1} \in \Omega$.

- White chooses a ball $W_{1} \in B_{1}^{\alpha}$.

- Black chooses a ball $B_{2} \in W_{1}^{\beta}$.

- And so on ad infinitum.

Finally, let $B_{i}^{*}=\psi\left(B_{i}\right)$ and $W_{i}^{*}=\psi\left(W_{i}\right)$. If $\bigcap_{i=1}^{\infty} B_{i}^{*}=\bigcap_{i=1}^{\infty} W_{i}^{*} \subseteq S$, then White wins the game. Otherwise, Black wins the game.

Our game can be understood in the following way. Initially, Black chooses a ball with radius $\rho_{1}$. Then, White chooses a ball with radius $\alpha \rho_{1}$ inside the first one. Now, Black chooses a ball with radius $\beta \alpha \rho_{1}$ inside the one chosen by White, and so on. In the end, the intersection of these balls will be non-empty by a simple corollary of Baire's Category Theorem. White wins the game if this intersection is a subset of $S$. Otherwise, Black wins.

Because of the topology of $\mathcal{L}^{m n}$, we may construct distinct elements $(\mathbf{c}, \rho),\left(\mathbf{c}^{\prime}, \rho^{\prime}\right) \in \Omega$ such that the corresponding balls in $\mathcal{L}^{m n}$ are the same, i.e., so that $\psi((\mathbf{c}, \rho))=\psi\left(\left(\mathbf{c}^{\prime}, \rho^{\prime}\right)\right)$ so that the map $\psi$ is not injective. However, we will often need to consider both the set $\psi((\mathbf{c}, \rho))$ and the formal ball $(\mathbf{c}, \rho)$ and will by abuse of notation denote both by

$$
\left\{\mathbf{x} \in \mathcal{L}^{m n}:\|\mathbf{x}-\mathbf{c}\|_{\infty} \leq \rho\right\},
$$

where $\mathbf{c}$ and $\rho$ are understood to be fixed, although changing these quantities could well have no effect on the set. 
The sets of particular interest to us are sets $S$ such that White can always win the $(\alpha, \beta ; S)$-game.

Definition. A set $S \subseteq \mathcal{L}^{m n}$ is said to be $(\alpha, \beta)$-winning for some $\alpha, \beta \in$ $(0,1)$ if White can always win the $(\alpha, \beta ; S)$-game. The set $S$ is said to be $\alpha$-winning for some $\alpha \in(0,1)$ if $S$ is $(\alpha, \beta)$-winning for any $\beta \in(0,1)$.

It is a fairly straightforward matter to see that if $S$ is $\alpha$-winning for some $\alpha$ and $\alpha^{\prime} \in(0, \alpha]$, then $S$ is $\alpha^{\prime}$-winning. Hence, we may define the maximal $\alpha$ for which a set is $\alpha$-winning.

Definition. Let $S \subseteq \mathcal{L}^{m n}$ and let $S^{*}=\{\alpha \in(0,1): S$ is $\alpha$-winning $\}$. The winning dimension of $S$ is defined as

$$
\text { windim } S= \begin{cases}0 & \text { if } S^{*}=\emptyset \\ \sup S^{*} & \text { otherwise. }\end{cases}
$$

We will first prove that the winning dimension of $\mathfrak{B}(m, n)$ is strictly positive. This will subsequently be used to deduce that the Hausdorff dimension of $\mathfrak{B}(m, n)$ is maximal. In order to do this, we study inequalities defined by slightly different matrices. For any $A \in \mathcal{L}^{m n}$, we define the matrices

$$
\widetilde{A}=\left(\begin{array}{cc}
A & I_{m} \\
I_{n} & 0
\end{array}\right), \quad \widetilde{A^{*}}=\left(\begin{array}{cc}
A^{T} & I_{n} \\
I_{m} & 0
\end{array}\right),
$$

where $I_{m}$ and $I_{n}$ denotes the $m \times m$ and $n \times n$ identity matrices respectively. Let $A^{(j)}$ denote the $j$ 'th column of the matrix $A$. In what follows, q will denote a vector in $\mathbb{F}[X]^{m+n}$ with coordinates $\mathbf{q}=\left(q_{1}, \ldots, q_{m+n}\right)$. Note that $A \in \mathfrak{B}(m, n)$ if and only if there exists a $K>0$ such that

$$
\max _{1 \leq j \leq n}\left(\left\|\mathbf{q} \cdot \widetilde{A}^{(j)}\right\|\right)^{n}>\frac{K}{\max _{1 \leq i \leq m}\left(\left\|q_{i}\right\|\right)^{m}}
$$

for any $\mathbf{q} \in \mathbb{F}[X]^{m+n}$ and such that the first $m$ coordinates of $\mathbf{q}$ are not all equal to zero.

These matrix inequalities allow us to examine the set $\mathfrak{B}(m, n)$ in terms of parallelepipeds in $\mathcal{L}^{m+n}$, i.e., sets defined by inequalities

$$
\left\|(\mathbf{x} A)_{i}\right\| \leq c_{i}, \quad A \in \mathcal{L}^{(m+n)^{2}}, c_{i}>0, i=1, \ldots, m+n,
$$

where $A$ is invertible and $(\mathbf{x} A)_{i}$ denotes the $i$ 'th coordinate of the vector $\mathbf{x} A$. Inspired by the theory of the geometry of numbers, we define distance functions

$$
F_{A}(\mathbf{x}):=\max _{1 \leq j \leq m+n} \frac{1}{c_{j}}\left\|\sum_{i=1}^{m+n} x_{i} a_{i j}\right\| .
$$

Also, for any $\lambda>0$, we define the sets

$$
P_{A}(\lambda)=\left\{\mathbf{x} \in \mathcal{L}^{m+n}: F_{A}(\mathbf{x}) \leq \lambda\right\} .
$$


Clearly, $P_{A}(1)$ is the set defined by (2.2). Also, for $\lambda^{\prime}<\lambda, P_{A}\left(\lambda^{\prime}\right) \subseteq P_{A}(\lambda)$.

In the setting of the real numbers, distance functions $F_{A}$ and sets $P_{A}$ are studied in the geometry of numbers (see [3] for an excellent account). For vector spaces over the field of Laurent series this theory was extensively developed by Mahler in [7]. We will only need a few results, which we summarise here.

Definition. Let $A \in \mathcal{L}^{(m+n)^{2}}$ be invertible. We define the $j^{\prime}$ th successive minimum $\lambda_{j}$ of $F_{A}$ to be

$$
\begin{aligned}
\lambda_{j}=\inf \left\{\lambda>0: P_{A}(\lambda) \text { contains } j\right. \text { linearly } \\
\left.\quad \text { independent (over } \mathcal{L}) \mathbf{a}_{1}, \ldots, \mathbf{a}_{j} \in \mathbb{F}[X]^{m+n}\right\} .
\end{aligned}
$$

We have the following lemma which is a corollary to the result in $[7$, Page 489]:

Lemma 2.1. For any invertible $A \in \mathcal{L}^{(m+n)^{2}}$,

$$
0<\lambda_{1} \leq \cdots \leq \lambda_{m+n}
$$

Furthermore,

$$
\lambda_{1} \cdots \lambda_{m+n}=\mu\left(P_{A}(1)\right)^{-1} .
$$

It should be noted that Mahler constructs the Haar measure in a different way from Sprindžuk's construction [11] used in [5]. However, as the Haar measure is unique up to a scaling factor, and since the measure of the unit $\|\cdot\|_{\infty}$-ball is equal to 1 in both constructions, the measures obtained in the two constructions must coincide.

It is easy to prove Theorem 1.1 from the above.

Proof of Theorem 1.1. Let $A \in \mathcal{L}^{m n}$. The Haar measure on $\mathcal{L}^{m+n}$ is invariant under linear transformations of determinant one (see [7]). Hence the measure of the set of $(\mathbf{x}, \mathbf{y}), \mathbf{x} \in \mathcal{L}^{m}, \mathbf{y} \in \mathcal{L}^{n}$ defined by the inequalities

$$
\|\mathbf{x} A-\mathbf{y}\|_{\infty} \leq q^{-Q m / n}, \quad\|\mathbf{x}\|_{\infty} \leq q^{Q},
$$

is equal to 1 , as it is obtained by such a transformation of the unit ball. The divisibility condition $n \mid Q m$ is used to ensure this. By Lemma 2.1, the first successive minimum of this set is less than or equal to 1 , so the set contains a non-trivial element $(\mathbf{q}, \mathbf{p}) \in \mathbb{F}[X]^{m+n}$. Furthermore, the inequalities imply that $\mathbf{q}$ cannot be trivial, which completes the proof.

We will need one additional result from [7, Page 489], relating the successive minima of a parallelepiped to those of its so-called polar body.

Lemma 2.2. Let $A \in \mathcal{L}^{(m+n)^{2}}$ be invertible, let $\lambda_{1}, \ldots, \lambda_{m+n}$ denote the successive minima of $F_{A}$ and let $\sigma_{1}, \ldots, \sigma_{m+n}$ denote the successive minima 
of the distance function $F_{A}^{*}$ defined by

$$
F_{A}^{*}(\mathbf{y})=\sup _{\mathbf{x} \neq 0} \frac{\|\mathbf{x} \cdot \mathbf{y}\|}{F_{A}(\mathbf{x})} .
$$

Then,

$$
\lambda_{m} \sigma_{n+1}=1 .
$$

The definition of a polar body can be taken to be the one implicit in the statement of Lemma 2.2, i.e.,

$$
P_{A}^{*}(\sigma)=\left\{\mathbf{x} \in \mathcal{L}^{m n}: F_{A}^{*}(x) \leq \sigma\right\} .
$$

This coincides with the definition used in [7, Chapter 5].

\section{The winning dimension of $\mathfrak{B}(\mathbf{m}, \mathbf{n})$}

In this section, we prove that the winning dimension of $\mathfrak{B}(m, n)$ is strictly positive. We will obtain an explicit lower bound on the winning dimension. For the rest of this section, let $n, m \in \mathbb{N}$ be fixed and $\alpha, \beta \in(0,1)$ be such that $\gamma=q^{-1}+\alpha \beta-\left(q^{-1}+1\right) \alpha>0$.

We now begin the game. Black starts by choosing a ball $B_{1}$ of radius $\rho=\rho\left(B_{1}\right)$. Clearly the set $B_{1}$ is bounded, so we may fix a $\sigma>0$ such that for all $A \in B_{1},\|A\|_{\infty} \leq \sigma$. We will construct a strategy for player White depending on a constant $R>R_{0}(\alpha, \beta, \rho, \sigma) \geq 1$, which we will choose later. We use subsequently

$$
\delta=R^{-m(m+n)^{2}}, \quad \delta^{*}=R^{-n(m+n)^{2}}, \quad \tau=\frac{m}{m+n} .
$$

Let $B_{k}, B_{h} \subseteq \mathcal{L}^{m n}$ be balls occurring in the $(\alpha, \beta)$-game chosen by Black such that $\rho\left(B_{k}\right)<R^{-(m+n)(\tau+i)}$ and $\rho\left(B_{h}\right)<R^{-(m+n)(1+j)}$ for some $i, j \in \mathbb{N}$. We will show that White can play in such a way that the following properties hold for $i, j \in \mathbb{N}$ :

- For $A \in B_{k}$, there are no $\mathbf{q} \in \mathbb{F}[X]^{m+n}$ such that the inequalities

$$
0<\max _{1 \leq l \leq m}\left\{\left\|q_{l}\right\|\right\} \leq \delta R^{n(\tau+i)}
$$

and

$$
\max _{1 \leq l^{\prime} \leq n}\left\{\left\|\mathbf{q} \cdot \widetilde{A}^{\left(l^{\prime}\right)}\right\|\right\} \leq \delta R^{-m(\tau+i)-n}
$$

both hold.

- For $A \in B_{h}$, there are no $\mathbf{q} \in \mathbb{F}[X]^{m+n}$ such that the inequalities

$$
0<\max _{1 \leq l^{\prime} \leq n}\left\{\left\|q_{l^{\prime}}\right\|\right\} \leq \delta^{*} R^{m(1+j)}
$$

and

$$
\max _{1 \leq l \leq m}\left\{\left\|\mathbf{q} \cdot \widetilde{A^{*}(l)}\right\|\right\} \leq \delta^{*} R^{-n(1+j)-m}
$$


both hold.

If a strategy such that (3.1a) and (3.1b) are avoided for all $i \in \mathbb{N}$ is followed, White will win the $(\alpha, \beta ; \mathfrak{B}(m, n))$-game. Indeed, given a $\mathbf{q} \in \mathbb{F}[X]^{m+n}$ with the first $m$ coordinates, $q_{1}, \ldots, q_{m}$ say, not all equal to zero, we can find an $i \in \mathbb{N}$ such that

$$
\delta R^{n(\tau+i-1)}<\max _{1 \leq l \leq m}\left\{\left\|q_{l}\right\|\right\} \leq \delta R^{n(\tau+i)} .
$$

This immediately implies that (3.1a) holds for this $i$, so that (3.1b) must be false. Hence, by (3.3),

$$
\begin{aligned}
\max _{1 \leq l^{\prime} \leq n}\left\{\left\|\mathbf{q} \cdot \widetilde{A}^{\left(l^{\prime}\right)}\right\|\right\}^{n} & >\frac{\delta^{m+n} R^{-m n(\tau+i)-n^{2}+m n(\tau+i)-m n}}{\max _{1 \leq l \leq m}\left\{\left\|q_{l}\right\|\right\}^{m}} \\
& =\frac{\delta^{m+n} R^{-n^{2}-m n}}{\max _{1 \leq l \leq m}\left\{\left\|q_{l}\right\|\right\}^{m}} \\
& >\frac{K}{\max _{1 \leq l \leq m}\left\{\left\|q_{l}\right\|\right\}^{m}}
\end{aligned}
$$

for any $K \in\left(0, \delta^{m+n} R^{-n^{2}-m n}\right)$, so the matrix $A$ is in $\mathfrak{B}(m, n)$ by (2.1).

For the remainder of this section, we will construct a strategy for White ensuring that (3.1a) and (3.1b) (resp. (3.2a) and (3.2b)) cannot hold for any $i$ (resp. $j$ ). We define for any $i \in \mathbb{N}$ :

- $B_{k_{i}}$ to be the first ball chosen by Black with $\rho\left(B_{k_{i}}\right)<R^{-(m+n)(\tau+i)}$.

- $B_{h_{i}}$ to be the first ball chosen by Black with $\rho\left(B_{h_{i}}\right)<R^{-(m+n)(1+i)}$.

Since $\tau<1$, these balls occur such that $B_{k_{0}} \supseteq B_{h_{0}} \supseteq B_{k_{1}} \supseteq B_{h_{1}} \supseteq \cdots$. By choosing $R$ large enough, we can ensure that the inclusions are proper.

Since

$$
\delta R^{n \tau}=R^{-m(m+n)^{2}+n m(m+n)^{-1}}=R^{-m\left((m+n)^{2}-\frac{n}{m+n}\right)}<1,
$$

(3.1a) has no solutions for $i=0$. Hence, White can certainly play in such a way that (3.1a) and (3.1b) have no polynomial solutions when $A \in B_{k_{0}}$. We will construct White's strategy in such a way that:

Step 1: Given the beginning of a game $B_{1} \supseteq W_{1} \supseteq \cdots \supseteq B_{k_{0}} \supseteq \cdots \supseteq B_{k_{i}}$ such that (3.1a) and (3.1b) have no polynomial solutions for any $A \in B_{k_{i}}$, White can play in such a way that (3.2a) and (3.2b) have no polynomial solutions for any $A \in B_{h_{i}}$.

Step 2: Given the beginning of a game $B_{1} \supseteq W_{1} \supseteq \cdots \supseteq B_{k_{0}} \supseteq \cdots \supseteq B_{h_{i}}$ such that (3.2a) and (3.2b) have no polynomial solutions for any $A \in B_{h_{i}}$, White can play in such a way that (3.1a) and (3.1b) have no polynomial solutions for any $A \in B_{k_{i+1}}$.

Our first lemma guarantees that we need only consider solutions to the equations in certain subspaces of $\mathcal{L}^{m+n}$. 
Lemma 3.1. Let $B_{1} \supseteq W_{1} \supseteq \cdots \supseteq B_{k_{i}}$ be the start of a game such that (3.1a) and (3.1b) have no polynomial solutions for any $A \in B_{k_{i}}$. The set

$$
\left\{\mathbf{q} \in \mathbb{F}[X]^{m+n}:(3.2 \mathrm{a}) \text { and }(3.2 \mathrm{~b}) \text { hold for } j=i \text { for some } A \in B_{k_{i}}\right\}
$$

contains at most $m$ linearly independent points.

Proof. Assume that there are linearly independent $\mathbf{q}_{1}, \ldots, \mathbf{q}_{m+1} \in \mathbb{F}[X]^{m+n}$ such that (3.2a) and (3.2b) hold for $A_{1}, \ldots, A_{m+1} \in B_{k_{i}}$. The absolute value of the first $n$ coordinates must be less than $\delta^{*} R^{m(1+i)}$ by (3.2a), and as $\left\|A_{u}\right\|_{\infty} \leq \sigma$ for $u=1, \ldots, m+1,(3.2 \mathrm{~b})$ and the structure of $\widetilde{A_{u}^{*}}$ guarantee that there is a constant $K_{1}(\sigma)>0$ such that

$$
\left\|\mathbf{q}_{u}\right\|_{\infty} \leq K_{1}(\sigma) \delta^{*} R^{m(1+i)} \quad \text { for } 1 \leq u \leq m+1 .
$$

Let $C$ be the centre of $B_{k_{i}}$. For any $A \in B_{k_{i}}$,

$$
\left\|\widetilde{A^{*}}(l)-{\widetilde{C^{*}}}^{(l)}\right\|_{\infty} \leq \rho\left(B_{k_{i}}\right)<R^{-(m+n)(\tau+i)} \quad \text { for } 1 \leq l \leq m .
$$

Now, as (3.2b) holds for the vectors $\mathbf{q}_{1}, \ldots, \mathbf{q}_{m+1}$, (3.4) and (3.5) imply that for $u=1, \ldots, m+1$,

$$
\begin{aligned}
& \max _{1 \leq l \leq m}\left\{\left\|\mathbf{q}_{u} \cdot{\widetilde{C^{*}}}^{(l)}\right\|\right\} \leq \max _{1 \leq l \leq m}\left\{\left\|\mathbf{q}_{u} \cdot \widetilde{A_{u}^{*}}(l)\right\|,\left\|\mathbf{q}_{u} \cdot\left(\widetilde{C^{*}}(l)-{\widetilde{A_{u}^{*}}}^{(l)}\right)\right\|\right\} \\
& \leq \max \left\{\delta^{*} R^{-n(1+i)-m}, K_{1}(\sigma) \delta^{*} R^{m(1+i)} R^{-(m+n)(\tau+i)}\right\} \\
& \leq K_{2}(\sigma) \delta^{*} R^{-n(1+i)},
\end{aligned}
$$

where $K_{2}(\sigma) \geq K_{1}(\sigma)>0$. If needed, we may increase the right hand side, so that without loss of generality, $K_{2}(\sigma)>1$.

We define the parallelepiped

$$
\begin{aligned}
& P=\left\{\mathbf{y} \in \mathcal{L}^{m+n}: \max _{1 \leq l^{\prime} \leq n}\left\{\left\|y l^{\prime}\right\|\right\} \leq R^{m(1+i)},\right. \\
&\left.\max _{1 \leq l \leq m}\left\{\left\|\mathbf{y} \cdot \widetilde{C^{*}}(l)\right\|\right\} \leq R^{-n(1+i)}\right\},
\end{aligned}
$$

along with the corresponding distance function $F_{C}$ and the successive minima $\lambda_{1}, \ldots, \lambda_{m+n}$. By (3.4) and (3.6), $\lambda_{m+1} \leq K_{2}(\sigma) \delta^{*}$. For $n=1,0<$ $\lambda_{m+1} \leq K_{2}(\sigma) R^{-(m+1)^{2}}$, which by Lemma 2.1 gives a contradiction by choosing $R$ large enough. 
Hence, we may assume that $n>1$. Let

$$
\begin{aligned}
& P^{*}=\left\{\mathrm{x} \in \mathcal{L}^{m+n}: \max _{1 \leq l \leq m}\left\{\left\|x_{l}\right\|\right\} \leq R^{n(1+i)},\right. \\
&\left.\max _{1 \leq l^{\prime} \leq n}\left\{\left\|\mathbf{x} \cdot \widetilde{C}^{\left(l^{\prime}\right)}\right\|\right\} \leq R^{-m(1+i)}\right\} .
\end{aligned}
$$

This set admits the distance function $F_{C}^{*}$ defined in Lemma 2.2 as the two bodies, $P$ and $P^{*}$, are mutually polar (see $[7]$ ).

Let $\sigma_{1}, \ldots, \sigma_{m+n}$ denote the successive minima of $P^{*}$. By Lemma 2.1 and Lemma 2.2,

$$
\begin{aligned}
\sigma_{1} \leq\left(\sigma_{1} \cdots \sigma_{n-1}\right)^{\frac{1}{n-1}}=\mu\left(P^{*}\right)^{\frac{-1}{n-1}}\left(\sigma_{n} \cdots \sigma_{m+n}\right)^{\frac{-1}{n-1}} \\
\leq \mu\left(P^{*}\right)^{\frac{-1}{n-1}} \sigma_{n}^{-\frac{m+1}{n-1}}=\mu\left(P^{*}\right)^{\frac{-1}{n-1}} \lambda_{m+1}^{\frac{m+1}{n-1}} \leq \mu\left(P^{*}\right)^{\frac{-1}{n-1}}\left(K_{2}(\sigma) \delta^{*}\right)^{\frac{m+1}{n-1}} \\
\quad \leq K_{3}(\sigma) R^{-(m+n)^{2}(m+1)}=K_{3}(\sigma) \delta R^{-(m+n)^{2}}
\end{aligned}
$$

where $K_{3}(\sigma)>0$. Hence, there is a $\mathbf{q} \in \mathbb{F}[X]^{m+n} \backslash\{0\}$ with

$$
\max _{1 \leq l \leq m}\left\{\left\|q_{l}\right\|\right\} \leq K_{3}(\sigma) \delta R^{-(m+n)^{2}} R^{n(1+i)}
$$

and

$$
\max _{1 \leq l^{\prime} \leq n}\left\{\left\|\mathbf{q} \cdot \widetilde{C}^{\left(l^{\prime}\right)}\right\|\right\} \leq K_{3}(\sigma) \delta R^{-(m+n)^{2}} R^{-m(1+i)}<1
$$

when we choose $R$ large enough. But by (3.7), $\max _{1 \leq l \leq m}\left\{\left\|q_{l}\right\|\right\}>0$, since otherwise the last $n$ coordinates would also be equal to 0 , whence $\mathbf{q}=0$. Hence, $R$ may be chosen large enough that $\mathbf{q}$ satisfies (3.1a) and (3.1b), a contradiction.

In a completely analogous way, we can prove:

Lemma 3.2. Let $B_{1} \supseteq W_{1} \supseteq \cdots \supseteq B_{h_{i}}$ be the start of a game such that (3.2a) and (3.2b) have no polynomial solutions for any $A \in B_{h_{i}}$. The set

$$
\begin{aligned}
\left\{\mathbf{q} \in \mathbb{F}[X]^{m+n}:(3.1 \mathrm{a})\right. & \text { and }(3.1 \mathrm{~b}) \\
& \text { hold with } \left.i \text { replaced by } i+1 \text { for some } A \in B_{h_{i}}\right\}
\end{aligned}
$$

contains at most $n$ linearly independent points.

We will now reduce the statement that White has a strategy such that Step 1 is possible to the statement that White can win a certain finite game. The converse Step 2 is analogous.

Once again, we assume that $B_{1} \supseteq W_{1} \supseteq \cdots \supseteq B_{k_{i}}$ is the beginning of a game such that we have avoided polynomial solutions to all relevant 
inequalities so far. It is sufficient for White to avoid solutions $\mathbf{q} \in \mathbb{F}[X]^{m+n}$ to $(3.2 \mathrm{a})$ and $(3.2 \mathrm{~b})$ with

$$
\delta^{*} R^{m(1+i-1)}<\max _{1 \leq l^{\prime} \leq n}\left\{\left\|q_{l^{\prime}}\right\|\right\} \leq \delta^{*} R^{m(1+i)},
$$

as solutions have been avoided for all vectors $\mathbf{q}$ with

$$
\max _{1 \leq l^{\prime} \leq n}\left\{\left\|q_{l^{\prime}}\right\|\right\} \leq \delta^{*} R^{m(1+i-1)}
$$

in the preceeding steps by assumption. Hence we need only consider the $\mathbf{q} \in \mathbb{F}[X]^{m+n}$ for which

$$
\delta^{*} R^{m(1+i-1)}<\|\mathbf{q}\|_{\infty} .
$$

By Lemma 3.1, the set of $\mathbf{q}$ satisfying (3.2a) and (3.2b) is contained in some $m$-dimensional subspace. Let $\left\{\mathbf{y}_{1}, \ldots, \mathbf{y}_{m}\right\}$ be a basis for this subspace, such that $\left\|\mathbf{y}_{i} \cdot \mathbf{y}_{i}\right\|=1$ for $1 \leq i \leq m$, and such that $\left\|\mathbf{y}_{i} \cdot \mathbf{y}_{j}\right\|=0$ whenever $i \neq j$. Note that this is possible if we require that the solutions $\mathbf{q}$ to be avoided are not in one of the finitely many one-dimensional linear subspaces of $\mathcal{L}^{m+n}$ in which the integer vectors satisfy $\mathbf{q} \cdot \mathbf{q}=0$. This in turn causes no loss of generality, since we can require the first ball chosen be Black to be bounded away from all these spaces, so that solutions within these spaces are automatically avoided. Write all $\mathbf{q}$ in this subspace satisfying (3.8) in the form $\mathbf{q}=t_{1} \mathbf{y}_{1}+\cdots+t_{m} \mathbf{y}_{m}, t_{1}, \ldots, t_{m} \in \mathcal{L}$. Immediately,

$$
\delta^{*} R^{m(1+i-1)}<\max _{1 \leq l^{\prime} \leq m}\left\{\left\|t_{l^{\prime}}\right\|\right\} .
$$

White needs to avoid solutions to the inequalities

$$
\max _{1 \leq l \leq m}\left\|\sum_{l^{\prime}=1}^{m} t_{l^{\prime}}\left(\mathbf{y}_{l^{\prime}} \cdot \widetilde{A^{*}}(l)\right)\right\| \leq \delta^{*} R^{-n(1+i)-m} .
$$

This matrix inequality may be solved using Cramer's Rule [6, Chapter XIII, Theorem 4.4]. This theorem shows that (3.10) is soluble only if for $l^{\prime}=1, \ldots, m$,

$$
\left\|t_{l^{\prime}}\right\|\|D\|=\left\|t_{l^{\prime}} D\right\| \leq \delta^{*} R^{-n(1+i)-m} \max _{1 \leq l \leq m}\left\{\left\|D_{l, l^{\prime}}\right\|\right\}
$$

where $D$ denotes the determinant of the matrix with entries $\mathbf{y}_{l^{\prime}} \cdot \widetilde{A^{*}}(l)$ and $D_{l, l^{\prime}}$ denotes the $\left(l, l^{\prime}\right)^{\prime}$ th co-factor of this determinant. By (3.9), it is sufficient to avoid

$$
\begin{aligned}
\|D\| \leq R^{-n(1+i)-m-m(1+i-1)} \max _{1 \leq l, l^{\prime} \leq m}\left\{\left\|D_{l, l^{\prime}}\right\|\right\} & \\
& =R^{-(m+n)(1+i)} \max _{1 \leq l, l^{\prime} \leq m}\left\{\left\|D_{l, l^{\prime}}\right\|\right\} .
\end{aligned}
$$

We define the following finite game: 
Definition. Let $\mathbf{y}_{1}, \ldots, \mathbf{y}_{m} \in \mathcal{L}^{m+n}$ be a set of vectors, such that $\left\|\mathbf{y}_{i} \cdot \mathbf{y}_{i}\right\|=1$ for $1 \leq i \leq m$, and such that $\left\|\mathbf{y}_{i} \cdot \mathbf{y}_{j}\right\|=0$ whenever $i \neq j$. Let $B \subseteq \mathcal{L}^{m n}$ be a ball with $\rho(B)<1$ such that for any $A \in B,\|A\|_{\infty} \leq \sigma$. Let $\mu>0$ and let $\alpha, \beta \in(0,1)$ with $q^{-1}+\alpha \beta-\left(q^{-1}+1\right) \alpha>0$. White and Black take turns according to the rules of the $(\alpha, \beta)$-game, choosing balls inside $B$, but the game terminates when $\rho\left(B_{t}\right)<\mu \rho(B)$. White wins the game if

$$
\|D\|>\rho(B) \mu \max _{1 \leq l, l^{\prime} \leq m}\left\{\left\|D_{l, l^{\prime}}\right\|\right\}
$$

for any $A \in B_{t}$.

If White can win the finite game for any $\mu \in\left(0, \mu^{*}\right)$ for some $\mu^{*}=$ $\mu^{*}(\alpha, \beta, \sigma)>0$, then White can guarantee that (3.11) does not hold for any $A \in B_{h_{i}}$. To see this, let $B=B_{k_{i}}$ and let

$$
\mu=\frac{R^{-(m+n)(1+i)}}{\rho(B)} \leq(\alpha \beta)^{-1} R^{-n} .
$$

Choosing $R$ large enough, this will be less than $\mu^{*}$. It remains to be shown, that such a $\mu^{*}$ exists. We will do this by induction.

Let $A \in \mathcal{L}^{m n}, v \in\{1, \ldots, m\}$ and $\left\{\mathbf{y}_{1}, \ldots, \mathbf{y}_{m}\right\}$ be the system of vectors from the definition of the finite game. By considering all possible choices of $1 \leq i_{1}<\cdots<i_{v} \leq m$ and $1 \leq j_{1}<\cdots<j_{v} \leq m$, we obtain $\left(\begin{array}{c}m \\ v\end{array}\right)^{2}$ matrices

$$
\left(\begin{array}{ccc}
\mathbf{y}_{i_{1}} \cdot \widetilde{A}^{*}\left(j_{1}\right) & \cdots & \mathbf{y}_{i_{1}} \cdot \widetilde{A^{*}}\left(j_{v}\right) \\
\vdots & & \vdots \\
\mathbf{y}_{i_{v}} \cdot \widetilde{A^{*}} & \cdots & \mathbf{y}_{i_{v}} \cdot \widetilde{A^{*}}\left(j_{v}\right)
\end{array}\right)
$$

For each $v \in\{1, \ldots, m\}$, we define the function $M_{v}: \mathcal{L}^{m n} \rightarrow \mathcal{L}^{\left(\begin{array}{c}m \\ v\end{array}\right)^{2}}$ to have as its coordinates the determinants of the matrices in (3.12) in some arbitrary but fixed order. Furthermore, define

$$
M_{-1}(A)=M_{0}(A)=(1),
$$

the standard unit vector in $\mathcal{L}^{\left(\begin{array}{c}m \\ 0\end{array}\right)^{2}}=\mathcal{L}$. For a set $K \subseteq \mathcal{L}^{m n}$, we define

$$
M_{v}(K)=\max _{A \in K}\left\|M_{v}(A)\right\|_{\infty} .
$$

We will prove a series of lemmas, culminating in a proof that under appropriate conditions, player White may always win the finite game (Lemma 3.5 below).

In the following, assume that $v>0$ and that there exists a $\mu_{v-1}$ such that

$$
\left\|M_{v-1}(A)\right\|_{\infty}>\rho(B) \mu_{v-1} M_{v-2}\left(B_{i_{v-1}}\right)
$$

for all $A \in B_{i_{v-1}}$ for an appropriate $B_{i_{v-1}}$ occurring in the game. 
Lemma 3.3. Let $\epsilon>0$ and let $B^{\prime} \subseteq B_{i_{v-1}}$ be a ball of radius

$$
\rho\left(B^{\prime}\right)<\epsilon \mu_{v-1} \rho\left(B_{i_{v-1}}\right) \text {. }
$$

Then

$$
\left\|M_{v-1}(A)-M_{v-1}\left(A^{\prime}\right)\right\|_{\infty}<\epsilon \rho(B) \mu_{v-1} M_{v-2}\left(B_{i_{v-1}}\right)
$$

for any $A, A^{\prime} \in B^{\prime}$.

Proof. Consider first for a fixed $A \in B^{\prime}$ and a fixed $x \in \mathcal{L}$ the quantity

$$
\left\|M_{v-1}\left(A+x E_{i j}\right)-M_{v-1}(A)\right\|_{\infty},
$$

where $E_{i j}$ denotes the matrix with 1 in the $i j$ 'th entry and zeros elsewhere. On considering an individual coordinate of the vector

$$
M_{v-1}\left(A+x E_{i j}\right)-M_{v-1}(A)
$$

and applying the ultra-metric inequality (1.2), it is seen that

$$
\left\|M_{v-1}\left(A+x E_{i j}\right)-M_{v-1}(A)\right\|_{\infty} \leq\|x\| M_{v-2}\left(B_{i_{v-1}}\right) .
$$

The factor $M_{v-2}\left(B_{i_{v-1}}\right)$ is an upper bound on the co-factor corresponding to the $i j$ 'th minor. When $\|x\|=1$, these quantities are discrete analogues of the partial derivatives of $M_{v-1}$, and the upper bound (3.14) implies that the function does not vary wildly.

We may pass from one matrix $A \in B^{\prime}$ to another $A^{\prime}$ by changing one coordinate at a time, i.e., by performing a string of $m n$ operations $A \mapsto$ $A+\left(A_{i j}^{\prime}-A_{i j}\right) E_{i j}$. Using these operations, we define a finite sequence of matrices by $A^{(1,1)}=A+\left(A_{11}^{\prime}-A_{11}\right) E_{11}, A^{(2,1)}=A^{(1,1)}+\left(A_{21}^{\prime}-A_{21}^{(1,1)}\right) E_{21}$ and so on, so that $A^{(m, n)}=A^{\prime}$. We now obtain,

$$
\begin{gathered}
\left\|M_{v-1}(A)-M_{v-1}\left(A^{\prime}\right)\right\|_{\infty}=\| M_{v-1}(A)-M_{v-1}\left(A^{(1,1)}\right)+M_{v-1}\left(A^{(1,1)}\right) \\
-\cdots-M_{v-1}\left(A^{((m-1), n)}\right)+M_{v-1}\left(A^{((m-1), n)}\right)-M_{v-1}\left(A^{\prime}\right) \|_{\infty}
\end{gathered}
$$

Here, each matrix in the arguments of $M_{v-1}$ differs from the preceding one in at most one place. Applying (3.14) and the ultra-metric inequality (1.2) $m n$ times,

$$
\begin{aligned}
\| M_{v-1}(A)- & M_{v-1}\left(A^{\prime}\right)\left\|_{\infty} \leq\right\| A-A^{\prime} \|_{\infty} M_{v-2}\left(B_{i_{v-1}}\right) \\
& <\epsilon \mu_{v-1} \rho\left(B_{i_{v-1}}\right) M_{v-2}\left(B_{i_{v-1}}\right) \leq \epsilon \rho(B) \mu_{v-1} M_{v-2}\left(B_{i_{v-1}}\right) .
\end{aligned}
$$

Corollary 3.1. For a ball $B^{\prime} \subseteq B_{i_{v-1}}$ with radius $\rho\left(B^{\prime}\right)<\frac{1}{2} \mu_{v-1} \rho\left(B_{i_{v-1}}\right)$, we have

for any $A^{\prime} \in B^{\prime}$.

$$
\left\|M_{v-1}\left(A^{\prime}\right)\right\|_{\infty}>\frac{1}{2} M_{v-1}\left(B^{\prime}\right)
$$


Proof. Apply Lemma 3.3 with $\epsilon=\frac{1}{2}$ and use (3.13).

Now, we define

$$
D_{v}(A)=\operatorname{det}\left(\begin{array}{ccc}
\mathbf{y}_{1} \cdot \widetilde{A}^{(1)} & \ldots & \mathbf{y}_{1} \cdot{\widetilde{A^{*}}}^{(v)} \\
\vdots & & \vdots \\
\mathbf{y}_{v} \cdot \widetilde{A}^{(1)} & \ldots & \mathbf{y}_{v} \cdot{\widetilde{A^{*}}}^{(v)}
\end{array}\right) .
$$

Clearly, this is a function of the $n v$ variables $a_{11}, \ldots, a_{n 1}, \ldots, a_{n v}$. We define the discrete gradient of $D_{v}$ to be the vector

$$
\nabla D_{v}(A)=\left(\begin{array}{c}
D_{v}\left(A+E_{11}\right)-D_{v}(A) \\
\vdots \\
D_{v}\left(A+E_{m n}\right)-D_{v}(A),
\end{array}\right) \in \mathcal{L}^{m n},
$$

where $E_{i j} \in \mathcal{L}^{m n}$ denotes the matrix having 1 as the $i j$ 'th entry and zeros elsewhere.

Corollary 3.2. With $B^{\prime}$ as in Lemma 3.3 and $A^{\prime}, A^{\prime \prime} \in B^{\prime}$, we have

$$
\left\|\nabla D_{v}\left(A^{\prime}\right)-\nabla D_{v}\left(A^{\prime \prime}\right)\right\|_{\infty} \leq K_{4}\left\|M_{v-1}\left(A^{\prime}\right)-M_{v-1}\left(A^{\prime \prime}\right)\right\|_{\infty}
$$

for some $K_{4}>0$ depending only on $m$ and $n$.

Proof. Note, that the coordinates of $\nabla D_{v}(A)$ are linear combinations of the coordinates of $M_{v-1}(A)$ for any $A$.

The discrete gradient of $D_{v}$ turns out to be the key ingredient in the proof. We will need the following lemma:

Lemma 3.4. Let $B^{\prime} \subseteq B_{i_{v-1}}$ be a ball such that

$$
\rho\left(B^{\prime}\right)<\frac{1}{2} \mu_{v-1} \rho\left(B_{i_{v-1}}\right) .
$$

Let $A^{\prime} \in B^{\prime}$ be such that

$$
\left\|M_{v}\left(A^{\prime}\right)\right\|_{\infty}<\frac{1}{8} M_{v-1}\left(B^{\prime}\right) .
$$

Furthermore, assume that the maximum $\left\|M_{v-1}\left(A^{\prime}\right)\right\|_{\infty}$ is attained by the absolute value of the coordinate which is the determinant

$$
d_{v}=\operatorname{det}\left(\begin{array}{ccc}
\mathbf{y}_{1} \cdot \widetilde{A^{\prime *}}(1) & \ldots & \mathbf{y}_{1} \cdot \widetilde{A^{\prime *}}(v-1) \\
\vdots & & \vdots \\
\mathbf{y}_{v-1} \cdot \widetilde{A^{\prime *}}(1) & \cdots & \mathbf{y}_{v-1} \cdot \widetilde{A^{* *}}(v-1)
\end{array}\right) .
$$

Then

$$
\left\|\nabla D_{v}\left(A^{\prime}\right)\right\|_{\infty}>K_{5}(\sigma) M_{v-1}\left(B^{\prime}\right)
$$

for some $K_{5}(\sigma)>0$. 
Proof. Let $\mathbf{z} \in \mathcal{L}^{m+n}$. Consider the following quantity

$$
\begin{aligned}
\Phi(\mathbf{z})= & \operatorname{det}\left(\begin{array}{rrr}
\mathbf{y}_{1} \cdot \widetilde{A^{\prime *}}(1) & \cdots & \mathbf{y}_{1} \cdot\left(\widetilde{A^{\prime *}}(v)\right. \\
\vdots & & \vdots \\
\mathbf{y}_{v} \cdot \widetilde{A^{\prime *}}(1) & \cdots & \mathbf{y}_{v} \cdot\left(\widetilde{A^{\prime *}}(v)\right. \\
& \cdots \mathbf{z})
\end{array}\right) \\
& -\operatorname{det}\left(\begin{array}{ccc}
\mathbf{y}_{1} \cdot \widetilde{A^{\prime *}}(1) & \cdots & \mathbf{y}_{1} \cdot \widetilde{A^{\prime *}}(v) \\
\vdots & & \vdots \\
\mathbf{y}_{v} \cdot \widetilde{A^{\prime *}}(1) & \cdots & \mathbf{y}_{v} \cdot \widetilde{A^{\prime *}}(v)
\end{array}\right) \| \\
= & \left\|\left(\sum_{h=1}^{v}(-1)^{h+1} d_{h} \mathbf{y}_{h}\right) \cdot \mathbf{z}\right\| .
\end{aligned}
$$

The last equality follows on expanding the determinants in the last column, where the $d_{i}$ are taken from the coordinates of $M_{v-1}\left(A^{\prime}\right)$ and $d_{v}$ is the special coordinate for which the maximum absolute value is attained.

Let $\mathbf{z}_{1}=d_{1} \mathbf{y}_{1}+\cdots+d_{v-1} \mathbf{y}_{v-1}+X d_{v} \mathbf{y}_{v}$, where $X \in \mathcal{L}$ is the power series consisting solely of the indeterminate $X$. We have assumed that $\left\|d_{i}\right\| \leq$ $\left\|d_{v}\right\|<q\left\|d_{v}\right\|=\left\|X d_{v}\right\|$ for all $i=1, \ldots, v-1$. Hence, by assumption on the $\mathbf{y}_{i}$,

$$
\begin{aligned}
\left\|\left(\sum_{h=1}^{v}(-1)^{h+1} d_{h} \mathbf{y}_{h}\right) \cdot \mathbf{z}_{1}\right\| & \\
=\|\left(\sum_{h=1}^{v}(-1)^{h+1} d_{h} \mathbf{y}_{h}\right) \cdot\left(d_{1} \mathbf{y}_{1}+\cdots+X d_{v} \mathbf{y}_{v}\right) & \| \\
& =q\left\|d_{v}\right\|^{2}=q\left\|M_{v-1}\left(A^{\prime}\right)\right\|_{\infty}^{2}>\frac{q}{4} M_{v-1}\left(B^{\prime}\right)^{2}
\end{aligned}
$$

by Corollary 3.1 .

We wish to interpret $\Phi(\mathbf{z})$ as a discrete analogue of the directional derivative along a vector in $\mathcal{L}^{m n}$. Furthermore, we need to obtain a lower bound on this quantity for some direction. In order to be able to make this interpretation, we need to find a lower bound on $\Phi\left(\mathbf{z}_{2}\right)$, where $\mathbf{z}_{2}$ is of the form $\left(z_{1}, \ldots, z_{n}, 0, \ldots, 0\right)$, i.e., where the last $m$ coordinates are zero. For such vectors, considering the difference in (3.17) corresponds to considering the difference $\left\|D_{v}\left(A+\hat{Z}_{2}\right)-D_{v}(A)\right\|$, where $\hat{Z}_{2} \in \mathcal{L}^{m n}$ is the matrix which has the vector $\left(z_{1}, \ldots, z_{n}\right)$ as its $v$ 'th row and zeros elsewhere, so that the matrix $A+\hat{Z}_{2} \in \mathcal{L}^{m n}$ is the matrix $A$ with the entries of the $v^{\prime}$ th row shifted by the first $n$ coordinates of $\mathbf{z}_{2}$. When $\left\|\hat{Z}_{2}\right\|_{\infty}=1$, this quantity is exactly the discrete directional derivative of $D_{v}$ in direction $\hat{Z}_{2}$ evaluated at $A$. 
Because of the special form of the $\widetilde{A^{\prime *}}(l)$, we may write

$$
\mathbf{y}_{h}=\mathbf{y}_{h}^{0}+\lambda_{h 1} \widetilde{A^{\prime *}}(1)+\cdots+\lambda_{h m} \widetilde{A^{\prime *}}(m),
$$

where the $\mathbf{y}_{h}^{0}$ have zeros on the last $m$ coordinates. By assumption on the $\mathbf{y}_{h}$, certainly for all $h, l,\left\|\lambda_{h, l}\right\| \leq 1$. Also, there is a constant $K_{5}(\sigma)>0$ such that $\left\|\mathbf{y}_{h}^{0}\right\|_{\infty} \leq \frac{1}{8} K_{5}(\sigma)^{-1}$ for all $h$. We define $\mathbf{z}_{2}=d_{1} \mathbf{y}_{1}^{0}+\cdots+d_{v-1} \mathbf{y}_{v-1}^{0}+$ $X d_{v} \mathbf{y}_{v}^{0}$, which clearly has the required form.

Now,

$$
\begin{aligned}
\Phi\left(\mathbf{z}_{2}\right) & =\left\|\left(\sum_{h=1}^{v}(-1)^{h+1} d_{h} \mathbf{y}_{h}\right) \cdot \mathbf{z}_{2}\right\| \\
& =\left\|\left(\sum_{h=1}^{v}(-1)^{h+1} d_{h} \mathbf{y}_{h}\right) \cdot\left(\mathbf{z}_{2}-\mathbf{z}_{1}+\mathbf{z}_{1}\right)\right\| \\
& \geq \frac{q}{4} M_{v-1}\left(B^{\prime}\right)^{2}-\left\|\left(\sum_{h=1}^{v}(-1)^{h+1} d_{h} \mathbf{y}_{h}\right) \cdot\left(\mathbf{z}_{1}-\mathbf{z}_{2}\right)\right\| .
\end{aligned}
$$

In order to produce a good lower bound for $\Phi\left(\mathbf{z}_{2}\right)$, we will produce a good upper bound on the last term of the above. We know that

$$
\mathbf{z}_{1}-\mathbf{z}_{2}=\sum_{l=1}^{m}\left(\sum_{h=1}^{v} d_{h}^{\prime} \lambda_{h l}\right){\widetilde{A^{\prime *}}}^{(l)},
$$

where $d_{h}^{\prime}=d_{h}$ for $h=1, \ldots, v-1$ and $d_{v}^{\prime}=X d_{v}$. Furthermore for $l=$ $1, \ldots, m$, by simple calculation,

$$
\begin{aligned}
& \left\|\left(\sum_{h=1}^{v}(-1)^{h+1} d_{h} \mathbf{y}_{h}\right) \cdot \widetilde{A^{\prime *}}(l)\right\| \\
& =\left\|\operatorname{det}\left(\begin{array}{cccc}
\mathbf{y}_{1} \cdot \widetilde{A^{\prime *}}(1) & \cdots & \mathbf{y}_{1} \cdot \widetilde{A^{\prime *}}(v-1) & \mathbf{y}_{1} \cdot \widetilde{A^{\prime *}}(l) \\
\vdots & & \vdots & \\
\mathbf{y}_{v} \cdot \widetilde{A^{\prime *}}(1) & \cdots & \mathbf{y}_{v} \cdot \widetilde{A^{\prime *}}(v-1) & \mathbf{y}_{v} \cdot \widetilde{A^{\prime *}}(l)
\end{array}\right)\right\| \\
& \\
& \quad \leq\left\|M_{v}\left(A^{\prime}\right)\right\|_{\infty}<\frac{1}{8} M_{v-1}\left(B^{\prime}\right)
\end{aligned}
$$

by choice of $A^{\prime}$. Hence, as $\left\|d_{h}^{\prime}\right\| \leq q M_{v-1}\left(B^{\prime}\right)$,

$$
\begin{aligned}
& \left\|\left(\sum_{h=1}^{v}(-1)^{h+1} d_{h} \mathbf{y}_{h}\right) \cdot\left(\mathbf{z}_{1}-\mathbf{z}_{2}\right)\right\| \\
& \quad=\left\|\sum_{l=1}^{m}\left(\sum_{h^{\prime}=1}^{v} d_{h^{\prime}}^{\prime} \lambda_{h^{\prime} l}\right)\left(\sum_{h=1}^{v}(-1)^{h+1} d_{h} \mathbf{y}_{h}\right) \cdot \widetilde{A^{\prime *}}(l)\right\|<\frac{q}{8} M_{v-1}\left(B^{\prime}\right)^{2} .
\end{aligned}
$$


Together with (3.18) this implies

$$
\Phi\left(\mathbf{z}_{2}\right)>\frac{q}{8} M_{v-1}\left(B^{\prime}\right)^{2} .
$$

We wish to use the discrete directional derivative to obtain a lower bound on the discrete gradient. Let $\mathbf{z} \in \mathcal{L}^{m+n}$ be some vector of the form $\left(z_{1}, \ldots, z_{n}, 0, \ldots, 0\right)$, so that $\mathbf{z}$ corresponds to a matrix $\hat{Z}_{v} \in \mathcal{L}^{m n}$ with $\left(z_{1}, \ldots, z_{n}\right)$ as its $v$ 'th row and zeros elsewhere. Suppose further that $\|\mathbf{z}\|_{\infty}=\left\|\hat{Z}_{v}\right\|_{\infty}=1$. It is simple to show that $\left\|\nabla D_{v}\left(A^{\prime}\right)\right\|_{\infty} \geq \Phi(\mathbf{z})$.

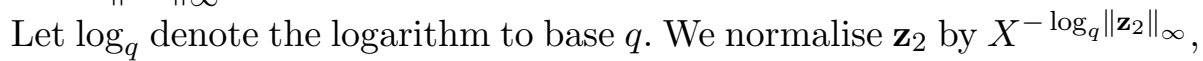
where $X$ is again the indeterminate in the power series expansions. In this way, we obtain a vector in $\mathcal{L}^{m+n}$ corresponding to a matrix $\hat{Z}_{2} \in \mathcal{L}^{m n}$ with $\left\|\hat{Z}_{2}\right\|_{\infty}=1$. Now, note that by (3.17), for any $x \in \mathcal{L}$ and any $\mathbf{z} \in \mathcal{L}^{m+n}$, $\Phi(x \mathbf{z})=\|x\| \Phi(\mathbf{z})$. But as $\left\|\mathbf{z}_{2}\right\|_{\infty} \leq \frac{1}{8} K_{5}(\sigma)^{-1} q M_{v-1}\left(B^{\prime}\right)$, we get by $(3.19)$

$$
\begin{gathered}
\left\|\nabla D_{v}(A)\right\|_{\infty} \geq \Phi\left(\mathbf{z}_{2} X^{\left.-\log _{q}\left\|\mathbf{z}_{2}\right\|_{\infty}\right)}=\frac{\Phi\left(\mathbf{z}_{2}\right)}{\left\|\mathbf{z}_{2}\right\|_{\infty}}\right. \\
>\frac{\frac{q}{8} M_{v-1}\left(B^{\prime}\right)^{2}}{\frac{1}{8} K_{5}(\sigma)^{-1} q M_{v-1}\left(B^{\prime}\right)}=K_{5}(\sigma) M_{v-1}\left(B^{\prime}\right) .
\end{gathered}
$$

This completes the proof.

We are now ready to prove that player White can win the finite game.

Lemma 3.5. Let $\left\{\mathbf{y}_{1}, \ldots, \mathbf{y}_{m}\right\}$ be a basis for this subspace, such that $\left\|\mathbf{y}_{i} \cdot \mathbf{y}_{i}\right\|=1$ for $1 \leq i \leq m$, and such that $\left\|\mathbf{y}_{i} \cdot \mathbf{y}_{j}\right\|=0$ whenever $i \neq j$. Let $B \subseteq \mathcal{L}^{m n}$ be a ball, $\rho(B)=\rho_{0}<1$, such that for some $\sigma>0,\|A\|_{\infty}<\sigma$ for any $A \in B$. Let $\alpha, \beta \in(0,1)$ with $q^{-1}+\alpha \beta-\left(q^{-1}+1\right) \alpha>0$. Assume that $0 \leq v \leq m$.

There exists a $\mu_{v}=\mu_{v}(\alpha, \beta, \sigma)>0$ for which White can play the finite game in such a way that for the first ball $B_{i_{v}}$ with $\rho\left(B_{i_{v}}\right)<\rho_{0} \mu_{v}$,

$$
\left\|M_{v}(A)\right\|_{\infty}>\rho_{0} \mu_{v} M_{v-1}\left(B_{i_{v}}\right)
$$

for any $A \in B_{i_{v}}$.

The slightly cumbersome notation $B_{i_{v}}$ is used in order to make the connection with (3.13), which we will use in the proof, explicit. Of course, the additional subscript plays no rôle in the statement of the lemma.

Proof. We will prove the lemma by induction. Clearly, the lemma holds for $v=0$. Hence, we use (3.13) as our induction hypothesis, and so we have the above results as our disposal.

Recall that $\gamma=q^{-1}+\alpha \beta-\left(q^{-1}+1\right) \alpha>0$ and let

$$
\epsilon=\frac{\gamma}{8} \frac{K_{5}(\sigma)}{K_{4}}>0 .
$$


Furthermore, let

$$
i_{v}=\min \left\{i \in \mathbb{N}: i>i_{v-1}, \rho\left(B_{i}\right)<\min \left(\frac{1}{2}, \epsilon\right) \mu_{v-1} \rho\left(B_{i_{v-1}}\right)\right\} .
$$

By appropriately choosing a constant $K_{6}(\alpha, \beta, \sigma)>0$, we have

$$
\rho\left(B_{i_{v}}\right) \geq K_{6}(\alpha, \beta, \sigma) \rho_{0}
$$

Using the induction hypothesis, Corollary 3.2 and Lemma 3.3, for any $A^{\prime}, A^{\prime \prime} \in B_{i_{v}}$, we have

$$
\left\|\nabla D_{v}\left(A^{\prime}\right)-\nabla D_{v}\left(A^{\prime \prime}\right)\right\|_{\infty}<K_{4} \epsilon \rho_{0} \mu_{v-1} M_{v-2}\left(B_{i_{v-1}}\right)<\frac{\gamma}{8} K_{5}(\sigma) M_{v-1}\left(B_{i_{v}}\right) .
$$

We now let

$$
\mu_{v}=\min \left\{\frac{1}{8}, \frac{\gamma}{8} \alpha \beta K_{6}(\alpha, \beta, \sigma), \frac{3 \gamma}{8} K_{5}(\sigma) K_{6}(\alpha, \beta, \sigma) K_{7}(\sigma)\right\}>0,
$$

where $K_{7}(\sigma)>0$ is to be chosen later. Assume that there exists an $A^{\prime} \in B_{i_{v}}$ for which the assertion of the lemma does not hold. That is,

$$
\left\|M_{v}\left(A^{\prime}\right)\right\|_{\infty} \leq \rho_{0} \mu_{v} M_{v-1}\left(B_{i_{v}}\right)
$$

In this case, we will prove that White has a strategy which will eliminate such elements in a finite number of moves.

By choice of $i_{v},(3.15)$ holds. Since $\rho_{0}<1,(3.16)$ holds. By rearranging the $\mathbf{y}_{i}$, we can without loss of generality assume that the condition on the determinant in Lemma 3.4 holds. Hence,

$$
\left\|\nabla D_{v}\left(A^{\prime}\right)\right\|_{\infty}>K_{5}(\sigma) M_{v-1}\left(B_{i_{v}}\right) .
$$

Let $\nabla^{\prime}=\nabla D_{v}\left(A^{\prime}\right)$, and let $D_{i}$ and $C_{i}$ denote the centres of $W_{i}$ and $B_{i}$ respectively. White can play in such a way that

$$
\left\|\left(C_{i}-D_{i}\right) \cdot \nabla^{\prime}\right\| \geq q^{-1}(1-\alpha) \rho\left(B_{i}\right)\left\|\nabla^{\prime}\right\|_{\infty} .
$$

Indeed, there are points $D_{i} \in B_{i}$ with $\left\|C_{i}-D_{i}\right\|_{\infty} \geq q^{-1}(1-\alpha) \rho\left(B_{i}\right)$ and such that $B\left(D_{i}, \alpha \rho\left(B_{i}\right)\right) \subseteq B_{i}$. This guarantees (3.23). Also, no matter how Black plays

$$
\left\|\left(C_{i+1}-D_{i}\right) \cdot \nabla^{\prime}\right\| \leq(1-\beta) \rho\left(W_{i}\right)\left\|\nabla^{\prime}\right\|_{\infty},
$$

since Black cannot choose the next centre further away from $D_{i}$. Hence,

$$
\begin{aligned}
&\left\|\left(C_{i+1}-C_{i}\right) \cdot \nabla^{\prime}\right\| \geq\left(q^{-1}(1-\alpha)-\alpha(1-\beta)\right) \rho\left(B_{i}\right)\left\|\nabla^{\prime}\right\|_{\infty} \\
&=\gamma \rho\left(B_{i}\right)\left\|\nabla^{\prime}\right\|_{\infty}>0 .
\end{aligned}
$$

We choose $t_{0} \in \mathbb{N}$ such that $\alpha \beta \frac{\gamma}{2}<(\alpha \beta)^{t_{0}} \leq \frac{\gamma}{2}$. Player White can ensure that

$$
\left\|\left(C_{i+t_{0}}-C_{i}\right) \cdot \nabla^{\prime}\right\| \geq \gamma \rho\left(B_{i}\right)\left\|\nabla^{\prime}\right\|_{\infty}>0 .
$$

This follows from (3.23), (3.24) and the fact that $\gamma>0$ so that player White can ensure that the bound in (3.25) is preserved for the next $t_{0}$ steps. White 
will play according to such a strategy. Furthermore $\rho\left(B_{i+t_{0}}\right) \leq \frac{\gamma}{2} \rho\left(B_{i}\right)$, so for any $A \in B_{i+t_{0}}$,

$$
\begin{aligned}
&\left\|\left(A-C_{i}\right) \cdot \nabla^{\prime}\right\| \geq\left\|\left(C_{i+t_{0}}-C_{i}\right) \cdot \nabla^{\prime}\right\|-\left\|\left(A-C_{i+t_{0}}\right) \cdot \nabla^{\prime}\right\| \\
& \geq \frac{\gamma}{2} \rho\left(B_{i}\right)\left\|\nabla^{\prime}\right\|_{\infty} .
\end{aligned}
$$

Now, for any $A \in B_{i_{v}}$,

$$
\begin{aligned}
&\left\|\left(A-C_{i_{v}}\right) \cdot \nabla D_{v}(A)\right\| \leq\left\|A-C_{i_{v}}\right\|_{\infty}\left\|\nabla D_{v}(A)\right\|_{\infty} \\
& \leq \rho\left(B_{i_{v}}\right) \max _{\substack{1 \leq l \leq m \\
1 \leq l^{\prime} \leq n}}\left\{\left\|D_{v}\left(A+E_{l l^{\prime}}\right)\right\|_{\infty},\left\|D_{v}(A)\right\|_{\infty}\right\} \leq K_{7}(\sigma)\left\|D_{v}(A)\right\|_{\infty}
\end{aligned}
$$

for some $K_{7}(\sigma)>0$. Also,

$$
\begin{array}{r}
\left\|\left(A-C_{i_{v}}\right) \cdot \nabla^{\prime}\right\|=\left\|\left(A-C_{i_{v}}\right) \cdot \nabla D_{v}(A)+\left(A-C_{i_{v}}\right) \cdot\left(\nabla^{\prime}-\nabla D_{v}(A)\right)\right\| \\
\leq \max \left\{\left\|\left(A-C_{i_{v}}\right) \cdot \nabla D_{v}(A)\right\|,\left\|\left(A-C_{i_{v}}\right) \cdot\left(\nabla^{\prime}-\nabla D_{v}(A)\right)\right\|\right\} \\
\leq\left\|\left(A-C_{i_{v}}\right) \cdot \nabla D_{v}(A)\right\|+\left\|\left(A-C_{i_{v}}\right) \cdot\left(\nabla D_{v}(A)-\nabla^{\prime}\right)\right\|
\end{array}
$$

Combining inequalities (3.28) and (3.29), we obtain for some $K_{7}(\sigma)>0$, (3.30)

$$
\left\|D_{v}(A)\right\|_{\infty} \geq K_{7}(\sigma)\left(\left\|\left(A-C_{i_{v}}\right) \cdot \nabla^{\prime}\right\|-\left\|\left(A-C_{i_{v}}\right) \cdot\left(\nabla D_{v}(A)-\nabla^{\prime}\right)\right\|\right) .
$$

Now by (3.27) and (3.22),

$$
\left\|\left(A-C_{i_{v}}\right) \cdot \nabla^{\prime}\right\| \geq \frac{\gamma}{2} \rho\left(B_{i_{v}}\right)\left\|\nabla^{\prime}\right\|_{\infty} \geq \frac{\gamma}{2} \rho\left(B_{i_{v}}\right) K_{5}(\sigma) M_{v-1}\left(B_{i_{v}}\right) .
$$

By (3.21),

$$
\begin{aligned}
\left\|\left(A-C_{i_{v}}\right) \cdot\left(\nabla D_{v}(A)-\nabla^{\prime}\right)\right\| \leq \rho\left(B_{i_{v}}\right) \| & \nabla D_{v}(A)-\nabla D_{v}\left(A^{\prime}\right) \|_{\infty} \\
& \leq \rho\left(B_{i_{v}}\right) \frac{\gamma}{8} K_{5}(\sigma) M_{v-1}\left(B_{i_{v}}\right) .
\end{aligned}
$$

Combining this with (3.30) and (3.31),

$$
\left\|D_{v}(A)\right\|_{\infty} \geq K_{7}(\sigma) \frac{3 \gamma}{8} \rho\left(B_{i_{v}}\right) K_{5}(\sigma) M_{v-1}\left(B_{i_{v}}\right) .
$$

Inserting (3.20) into this expression, we find that

$$
\left\|D_{v}(A)\right\|_{\infty} \geq \frac{3 \gamma}{8} K_{5}(\sigma) K_{6}(\alpha, \beta, \sigma) K_{7}(\sigma) \rho_{0} M_{v-1}\left(B_{i_{v}}\right) \geq \mu_{v} \rho_{0} M_{v-1}\left(B_{i_{v}}\right),
$$

by choice of $\mu_{v}$. This completes the proof of Lemma 3.5.

Note that Lemma 3.5 immediately implies:

Theorem 3.1. Let $\alpha, \beta \in(0,1)$ with $q^{-1}+\alpha \beta-\left(q^{-1}+1\right) \alpha>0$ and let $m, n \in \mathbb{N}$. White can win the finite game and hence the $(\alpha, \beta ; \mathfrak{B}(m, n))$ game. In particular,

$$
\operatorname{windim}(\mathfrak{B}(m, n)) \geq \frac{1}{q+1}
$$


Proof. The first part follows from Lemma 3.5 with $v=m$ and the obvious analogue for the other step in the strategy for the $(\alpha, \beta ; \mathfrak{B}(m, n))$-game. The lower bound follows as $q^{-1}+\alpha \beta-\left(q^{-1}+1\right) \alpha>0$ for any $\beta \in(0,1)$ and any $\alpha<1 /(q+1)$.

\section{The Hausdorff dimension of $\mathfrak{B}(\mathbf{m}, \mathbf{n})$}

In this final section, we will prove that if $\alpha>0$, then any $\alpha$-winning set in $\mathcal{L}^{m n}$ has maximal Hausdorff dimension. By Theorem 3.1, this will imply Theorem 1.2. To do this, we change our viewpoint to that of player Black. We will for each step of the game examine a number of different possible directions for the game under the assumption that player White is following a winning strategy. This will give rise to a particularly rich subset of the $\alpha$-winning set for which we may estimate the Hausdorff dimension.

Theorem 4.1. Let $\beta \in(0,1)$ and let $N(\beta) \in \mathbb{N}$ be such that any ball $B \subseteq \mathcal{L}^{m n}$ of radius $\rho$ contains $N(\beta)$ pairwise disjoint balls of radius $\beta \rho$. Let $S \subseteq \mathcal{L}^{m n}$ be $(\alpha, \beta)$-winning. Then

$$
\operatorname{dim}_{\mathrm{H}}(S) \geq \frac{\log N(\beta)}{|\log \alpha \beta|} .
$$

Proof. Let $\Lambda=\{0, \ldots, N(\beta)-1\}^{\mathbb{N}}$ and let $\left(i_{j}\right) \in \Lambda$. For each ball $W_{j}$ chosen by White, we pick $N(\beta)$ disjoint balls in $W_{j}^{\beta}$ which we enumerate by elements from the set $\{0, \ldots, N(\beta)-1\}$. We restrict the choice of moves for player Black to these $N(\beta)$ possibilities. In this way, we obtain for each element $\lambda \in \Lambda$ a point $A(\lambda) \in \mathcal{L}^{m n}$. As we may assume that White is following a winning strategy, for each $\lambda \in \Lambda, A(\lambda) \in S$. We will label balls chosen by player Black by the sequence leading to them, i.e., $B_{l}=$ $B\left(i_{1}, \ldots, i_{l}\right)$, where $i_{1}, \ldots, i_{j} \in\{0, \ldots, N(\beta)-1\}$. As distinct sequences give rise to disjoint balls from some point in the game and onwards, distinct points $\lambda, \lambda^{\prime} \in \Lambda$ give rise to different points $A(\lambda), A\left(\lambda^{\prime}\right) \in S$.

Let

$$
S^{*}=\bigcup_{\lambda \in \Lambda}\{A(\lambda)\} \subseteq S .
$$

We define a surjective function $f: S^{*} \rightarrow[0,1]$ by

$$
A \mapsto x=0 . i_{1} i_{2} \ldots \quad \text { where } A=A\left(i_{1}, i_{2}, \ldots\right)
$$

where $0 . i_{1} i_{2} \ldots$ is the base $N(\beta)$ expansion of $x$. We extend this function to all subsets of $\mathcal{L}^{m n}$ in the following way. For $T \subseteq S^{*}$, let $f(T)=\bigcup_{A \in T} f(A)$. For $R \subseteq \mathcal{L}^{m n}$, let $f(R)=f\left(R \cap S^{*}\right)$.

Let $\mathcal{C}=\left(B_{l}\right)_{l \in \mathbb{N}}$ be a cover of $S$ with balls, where $B_{l}$ has radius $\rho_{l}$. Clearly, $\mathcal{C}^{*}=\left(B_{l} \cap S^{*}\right)_{l \in \mathbb{N}}$ is a cover of $S^{*}$. Mapping to the interval, we find that $f\left(\mathcal{C}^{*}\right)=\left(f\left(B_{l} \cap S^{*}\right)\right)_{l \in \mathbb{N}}=\left(f\left(B_{l}\right)\right)_{l \in \mathbb{N}}$ is a cover of $[0,1]$. Thus, the 
union of the sets $f\left(B_{l}\right)$ has outer Lebesgue measure $\ell$ greater than 1 , so by sub-additivity

$$
\sum_{l=1}^{\infty} \ell\left(f\left(B_{l}\right)\right) \geq 1
$$

Now, let

$$
j_{l}=\left[\frac{\log 2 \rho_{l}}{\log \alpha \beta}\right] .
$$

For $\rho_{l}$ sufficiently small, we have $j_{l}>0$ and $\rho_{l}<(\alpha \beta)^{j_{l}}$. Hence, by the ball intersection property, $B_{l}$ is contained in at most one ball of the form $B_{l}\left(i_{1}, \ldots, i_{j_{l}}\right)$. But such a ball clearly maps into an interval of length $N(\beta)^{-j_{l}}$. Hence, $\ell\left(f\left(B_{l}\right)\right) \leq N(\beta)^{-j_{l}}$. By (4.1), we have

$$
\begin{aligned}
1 \leq \sum_{l=1}^{\infty} \ell\left(f\left(B_{l}\right)\right) \leq \sum_{l=1}^{\infty} N(\beta)^{-j_{l}} & \\
& =\sum_{l=1}^{\infty} N(\beta)^{-\left[\frac{\log 2 \rho_{l}}{\log \alpha \beta}\right]} \leq 2^{\frac{\log N(\beta)}{|\log \alpha \beta|}} \sum_{l=1}^{\infty} \rho_{l}^{\frac{\log N(\beta)}{|\log \alpha \beta|}} .
\end{aligned}
$$

Now, for any such cover $\mathcal{C}$ with small enough balls, the $s$-length $l^{s}(\mathcal{C})>0$ for $s=\frac{\log N(\beta)}{|\log \alpha \beta|}$. Hence,

$$
\operatorname{dim}(S) \geq \frac{\log N(\beta)}{|\log \alpha \beta|}
$$

Theorem 4.1 allows us to prove that $\operatorname{dim}(\mathfrak{B}(m, n))=m n$ and thus complete the proof of Theorem 1.2:

Proof of Theorem 1.2. By Theorem 4.1, we need only estimate the number $N(\beta)$ to get a lower bound for the Hausdorff dimension. This is a simple combinatorial problem. By scaling and translation, we note that it suffices to consider the $\|\cdot\|_{\infty}$-ball $B(0,1)=I^{m n}$.

We choose the number $i \in \mathbb{Z}$ such that $q^{i-1} \leq \beta<q^{i}$ and consider the balls $B(c, \beta) \subseteq I^{m n}$ where $c \in X^{i+1} \mathbb{F}[X]^{m n}$. By choice of $i$ and the ball intersection property, these are clearly disjoint. Furthermore, counting these balls we see that

$$
N(\beta)=\left(q^{-i-1}\right)^{m n}=\frac{1}{q^{m n}} \frac{1}{\left(q^{i}\right)^{m n}} \asymp \frac{1}{\beta^{m n}} .
$$

Hence, by Theorem 4.1,

$$
\operatorname{dim}(\mathfrak{B}(m, n)) \geq \frac{m n|\log \beta|}{|\log \alpha|+|\log \beta|} \underset{\beta \rightarrow 0}{\longrightarrow} m n .
$$

This completes the proof. 
Acknowledgements. I thank my Ph.D. supervisors R. Nair and M. Weber for their encouragement. In particular, I thank R. Nair for suggesting the topic of this paper. I also thank M. M. Dodson for his comments on the manuscript and an anonymous referee for his helpful remarks.

The bulk of the research presented in this paper was done while I was a student at the University of Liverpool. Many changes and corrections to the paper have been made since then. 


\section{References}

[1] A. G. Abercrombie, Badly approximable p-adic integers. Proc. Indian Acad. Sci. Math. Sci. 105 (2) (1995), 123-134.

[2] M. Amou, A metrical result on transcendence measures in certain fields. J. Number Theory 59 (2) (1996), 389-397.

[3] J. W. S. CAssels, An introduction to the geometry of numbers. Springer-Verlag, Berlin, 1997.

[4] V. JARník, Zur metrischen Theorie der diophantischen Approximationen. Prace Mat.-Fiz. (1928-1929), 91-106.

[5] S. Kristensen, On the well-approximable matrices over a field of formal series. Math. Proc. Cambridge Philos. Soc. 135 (2) (2003), 255-268.

[6] S. Lang, Algebra (2nd Edition). Addison-Wesley Publishing Co., Reading, Mass., 1984.

[7] K. MAhler, An analogue to Minkowski's geometry of numbers in a field of series. Ann. of Math. (2) 42 (1941), 488-522.

[8] H. Niederreiter, M. Vielhaber, Linear complexity profiles: Hausdorff dimensions for almost perfect profiles and measures for general profiles. J. Complexity 13 (3) (1997), 353383.

[9] W. M. Schmidt, On badly approximable numbers and certain games. Trans. Amer. Math. Soc. 123 (1966), 178-199.

[10] W. M. Sснмidt, Badly approximable systems of linear forms, J. Number Theory 1 (1969), 139-154.

[11] V. G. SprindžUK, Mahler's problem in metric number theory. American Mathematical Society, Providence, R.I., 1969.

\section{Simon Kristensen}

Department of Mathematical Sciences

Faculty of Science

University of Aarhus

Ny Munkegade, Building 530

8000 Aarhus C, Denmark

E-mail: sik@imf.au.dk 